\title{
Lusioersily
}

\section{Challenges to conducting randomised controlled trials with adults with intellectual disabilities: Experiences of international experts}

Mulhall, P., Taggart, L., McAloon, T., \& Coates, V. (2021). Challenges to conducting randomised controlled trials with adults with intellectual disabilities: Experiences of international experts. Journal of Applied Research in Intellectual Disabilities, 34(3), 891-904. https://doi.org/10.1111/jar.12838

Link to publication record in Ulster University Research Portal

Published in:

Journal of Applied Research in Intellectual Disabilities

Publication Status:

Published (in print/issue): 30/05/2021

DOI:

10.1111/jar.12838

Document Version

Author Accepted version

\section{General rights}

Copyright for the publications made accessible via Ulster University's Research Portal is retained by the author(s) and / or other copyright owners and it is a condition of accessing these publications that users recognise and abide by the legal requirements associated with these rights.

\section{Take down policy}

The Research Portal is Ulster University's institutional repository that provides access to Ulster's research outputs. Every effort has been made to ensure that content in the Research Portal does not infringe any person's rights, or applicable UK laws. If you discover content in the Research Portal that you believe breaches copyright or violates any law, please contact pure-support@ulster.ac.uk. 
Challenges to conducting randomised controlled trials with adults with intellectual disabilities: interviews with international experts.

Running Title: Challenges conducting ID-RCTs: experts' views

\section{Authors:}

Peter Mulhall*

Dr Laurence Taggart*

Dr Toni McAloon*

Prof Vivien Coates*

* School of Nursing, Ulster University, Newtownabbey, Northern Ireland

Corresponding author:

Peter Mulhall

School of Nursing

Ulster University

Shore Road

Newtownabbey

Co. Antrim

BT37 0JB

Northern Ireland

mulhall-p@ulster.ac.uk

00442890366318

Funding: This project was supported through a PhD funded by the Department of Education and Learning (DEL), Northern Ireland.

Conflicts of Interest: None 
Challenges to conducting randomised controlled trials with adults with intellectual disabilities: experiences of international experts.

Running Title: Challenges conducting ID-RCTs: experts' views

Keywords (phrases):

Intellectual Disability

Randomised Controlled Trials (RCTs);

Barriers and challenges;

Identification, consent and recruitment;

Ethical approval;

Attitudes towards ID RCTs.consort 


\title{
Challenges to conducting randomised controlled trials with adults with intellectual disabilities: experiences of international experts.
}

\begin{abstract}
Background: Globally, conducting randomised controlled trials can be a complex endeavour. The complexity increases when including participants with cognitive or intellectual disabilities. A fuller understanding of the barriers and challenges that can be expected in such trials may help researchers to make their trials more inclusive for people with disabilities.
\end{abstract}

Method: Semi-structured interviews were conducted with twelve international trials experts.

Results: Eight themes emerged relating to challenges linked to: 1) participant co-morbidities, 2) participant ability levels, 3) ethics and consent, 4) the RCT methodology, 5) gatekeeping, 6) staff turnover, 7) lack of technical understanding and 8) attitudes and perceptions.

Conclusion: Conducting trials with cognitively disabled participants can pose unique challenges although many can be overcome with 'reasonable adjustments'. Challenges that are harder to overcome are attitudes and perceptions that people (professional staff, funding bodies, carers or fellow researchers) hold towards the utility of conducting trials with cognitively disabled populations.

Abstract 149 words

Paper 7660 words (including quotes) 


\section{Introduction}

Randomised Controlled Trials (RCTs) are the most popular method for assessing treatment effectiveness within health provision research. A strength of the RCT methodology is the adherence to a pre-defined study protocol relating to recruitment, intervention and analysis. Thus, RCTs are said to have high levels of internal validity (Katkade et al., 2018). Since the rise of the RCT methodology, there has been criticism of the external validity of RCTs. Most of this criticism has centred on a) ethical considerations regarding randomising to control groups (Oakley et al., 2003), and b) the generalisability of results to 'real world' situations due to factors including small sample size, poor definition of syndromes, and heterogeneity of samples (Mulder et al., 2018). However, a range of adaptations have been made to the RCT design in order to test effectiveness in a myriad of practical realistic conditions (Brown et al. 2017).

In addition to practical and methodological threats, a trial's validity can also be negatively impacted by 'human factors'. There is growing research concerning the profile of participants who are often disproportionately included, and disproportionately excluded, from mainstream clinical trials (i.e. non disability-specific trials conducted in the general population). Bonevski et al. (2014) reported that in western health-related trials, white, middle-aged, middle-class males have been overrepresented in research samples. Heiat et al. (2002) in a review of heart failure RCTs found that women, minority groups, and the elderly were routinely excluded. This is surprising given that heart failure is more common among older people. Similarly, Scheifes et al. (2011) conducted a review of mainstream RCTs which tested the use of anti-psychotic medication for managing behavioural problems. Anti-psychotics are commonly prescribed to people with intellectual disabilities (ID) (Tyrer et al., 2008), and yet Scheifes et al. (2011) found that 73 of 100 trials excluded people with ID. Feldman et al. (2014) in a review of 300 randomly selected mainstream trials found that only $2 \%$ explicitly included people with ID, and $90 \%$ excluded 
people with ID, although neither Scheifer et al. (2011) nor Feldman et al. (2014) surveyed the trials authors about reasons for excluding people with ID. If RCTs are to have maximum external validity, then a crucial design factor is the inclusion of participants who match those most likely to receive the interventions being tested.

ID-RCTs: In order to encourage and empower researchers to include underrepresented sections of society into a study, it would be beneficial to have a thorough understanding of the barriers to inclusion. Each group of underrepresented people may have their own nuanced needs and challenges. However, it is conceivable that some challenges may be common across a number of sub-groups. A number of reviews have investigated the barriers to participation in clinical trials for certain underrepresented populations (Ford et al., 2008; Glover et al., 2015; Kadam et al., 2016) however these reviews have focused on populations based upon race and ethnicity. Only one systematic review has been conducted investigating reported challenges and barriers to conducting trials in the field of cognitive disabilities (Mulhall et al., 2018).

Within the cognitive disability field, it is widely acknowledged that people with ID die prematurely and have poorer health than their non-disabled peers on a range of health markers (Cooper, 2017). It is arguable that the evidence base for many ID clinical interventions, whether pharmacological or psychosocial, is at a very early stage of development, and has many gaps. Systematic reviews have consistently noted a lack of RCTs in the ID literature (Vereenooghr et al., 2013; Koslowski et al., 2016; Jones \& Chaplin, 2017). Moreover, many of the reported RCTs are under-powered, do not specify how they handled missing data and have used outcome measures which have not yet been validated with ID populations (Mulhall et al., 2018). It should be noted however that the last five years have seen an increase in the number of pragmatic trials within the Intellectual Disability (ID) field (Tyrer et al., 2017; Hassiotis et al., 2018; Jahoda et al., 
2018; McGill et al., 2018, Hunter et al., 2020) and the broader cognitive disability field (Olsson et al., 2017; Williams et al., 2020) wherein interventions are tested in situations more similar to routine clinical practice (Roland et al., 1998). The rise of pragmatic trials in the ID field is, arguably, still a recent phenomenon, and it has been proposed that research within the ID field often lags well behind other fields (Hastings, 2013).

Known Challenges and Barriers: A number of papers have highlighted challenges faced by authors whilst conducting specific ID-RCTs. These challenges include: identification of potential participants, staff work load and complex organisational structures (Lennox et al., 2005); excessive governance structures and unnecessary bureaucracy (Leeson \& Tyrer, 2013); heterogeneity of populations, and a lack of sensitive outcome measures (Jacquerment et al., 2014); difficulties obtaining consent, needing to rely on the good will of organisations, and agency fear of 'outsiders' (Siegel \& Ellis, 1985 - cited in Lennox et al., 2005).

In a recent systematic review of the adult ID RCT literature, Mulhall et al. (2018) found that methodological and practical barriers/challenges commonly reported in trials papers include: staff turnover; lack of disability-specific outcome measures; the need to adapt intervention programs and resources; and participant attrition/missing data. They also noted that a number of expected issues were not cited within the trials' papers. For example, none of the trials reviewed specifically noted the identification of participants as being a barrier, nor did they discuss negative staff attitudes towards research as having had an impact on their trial. Similarly, none of the reviewed papers cited the process of obtaining informed consent from people with intellectual or communication difficulties as posing a challenge. 
The Mulhall et al. (2018) review posed a number of pertinent questions about why there is a mismatch between the methodological and practical challenges that were reported in the wider literature and the challenges that were reported in the 'trials' papers. This point is important because it relates to the scientific principle of replication. Replication is essential to the development of scientific knowledge (Camerer et al., 2018). However, the ability to reproduce study findings in a number of fields has been questioned (Prinz et al., 2011; Button et al., 2013; Freedman et al., 2015). It could be argued that to date, the pursuit of replication has focused largely on statistical replication of results, with less than favourable success in many fields. This raises a fundamental question: how can the results of a trial be replicated if the actual trial itself cannot be replicated? How can a trial be replicated if we do not know what challenges and hurdles were faced, and how they were overcome? Thus, if replication is to be truly achievable then knowledge concerning the 'process' of conducting a trial is equally as important as the 'results' of the trial. Mulhall et al. (2018), in their systematic review, noted that trials reports add valuable contributions to the development of an 'evidence base' but often miss valuable opportunities to contribute to the 'experience base' within the ID field. Given that ID RCTs are still at an early stage of development, Mulhall and colleagues proposed that the development, and sharing, of the 'experience-base' is crucial to both encouraging non-disability researchers to include people with ID and other cognitive disabilities into their trials, and also to the further development of IDspecific trials.

If the necessary intervention evidence base in the ID field is to be built upon, then researchers interested in conducting ID-RCTs, or who wish to enable mainstream trials to be more inclusive and accessible, need a clearer understanding of the range of methodological and practical challenges they may face when either including people with ID in their trials or when conducting 
ID-specific trials. Limited research has begun to explore this. Robotham et al. (2011) explored barriers and the social validity of ID-RCTs from the perspectives of a number of stakeholders (service users, carers and professional staff) however, to date, the experiences and views of IDRCT researchers themselves do not appear have to be investigated, collated and reported.

The Medical Research Council (MRC) have produced guidelines to encourage researchers to share their 'practical' experiences conducting trials through the reporting of 'process evaluations' (Moore et al., 2015) (e.g. Bosco et al., 2019). The extent to which this aids the promotion and development of additional trials in this field may depend upon how often funders require process evaluations to be conducted and how often journal editors encourage their publication. It may also depend not just upon 'What' knowledge is shared, but also 'How' the knowledge is shared. It is well recognised that the possession of knowledge does not in itself guarantee change in behavioural practice (Duncombe, 2017). The science of Dissemination and Implementation has developed to "improve the adoption, appropriate adaptation, delivery, and sustainment of effective interventions" (Brown et al., 2017). Within this science, a wealth of models and frameworks have been proposed to aid the integration of scientifically collated knowledge into decision making and behavioural change.

This paper reports on part of a larger, multi-stage sequential research program which uses an adapted version of Aarons et al. (2011) model for evidence-based practice implementation. Aarons et al. (2011) proposed a fourphase model: Exploration, Adoption Decision/Preparation, Implementation, Sustainment. The Aaron et al. model is particularly applicable to this study as it is aimed at public sector implementation, and at each phase focuses on a range of 'Inner' and 'Outer' contextual factors including socio-political factors such as legislation, policies, funding, client advocacy, organisational and individual characteristics. In relation to our larger program of 
study, the Exploration phase refers to an awareness that ID-RCT researchers face a range of challenges, some are common with other fields and some are unique to participants with ID. The Adoption Decision refers to an acknowledgement of the presence of practical and methodological barriers in ID-RCTs, that these may be influenced by a range of factors, and the utility of sharing this knowledge. Both of these phases can be seen as being reported in the Mulhall et al. (2018) systematic review on this topic. The Preparation refers to the development of a mechanism for collating the knowledge (taking account of the range of influencing factors), and establishing a network for sharing the knowledge (this is reported in the current paper and further developed using a Delphi Survey (ANON, 2020)). The Implementation and Sustainment phases refer to the development of an online resource toolkit which not only allows for the sharing of the knowledgebase to a range of audiences, but also for the continued collating and sharing on an ongoing and free to access basis. This will be presented in a future paper.

\begin{abstract}
Aim
The aim of this study was to explore the range of methodological and practical challenges faced by researchers conducting RCTs with adults with ID.
\end{abstract}

\title{
Methods
}

This study employed a qualitative methodology utilising a series of one-to-one semi-structured interviews.

\section{Participants and sampling}

Two sampling approaches were used with this study. A purposive sampling frame was drawn from a set of primary authors of papers included in a systematic review detailed in Mulhall et al. 
(2018). Opportunity sampling was used to identify potential interviewees via the authors' academic network. A total of 34 invitations were sent and twelve participants consented to participate. All interviewees were experienced ID researchers.

Twelve researchers (six males and six females) from a range of professional and research backgrounds took part in this study. Professional backgrounds included: Psychiatry; Psychology; Medicine; Public Health; Professions Allied to Medicine (PAM); and Academia. Participants were from the United Kingdom $(n=6)$, Australia $(n=2)$, Sweden $(n=2)$, Spain $(n=1)$ and the USA (1). The average length of time researching in this field was 16 years $(\min =10, \max =35)$ and the average number of trials conducted per interviewee in this field was $3(\min =1, \max =8)$.

\section{Interview format}

A semi-structured interview schedule was developed that was informed by the existing ID-RCT literature, coupled with the results of a systematic review of the methodological challenges (Mulhall et al., 2018). The interview schedule comprised of a series of open-ended questions covering seven main areas: 1) trial planning, 2) funding $\&$ funding bodies, 3) ethical approval \& consent, 4) recruitment, 5) conducting the trial (e.g. maintaining fidelity to protocol), 6) analysis and 7) reporting. These topic areas were used to give initial structure to the interventions, they were not used in the coding and analysis of the data, and are not used in the presentation of the findings below. Each interview had five main phases, as per guidance from Robson (2011): introduction; warm up questions; main questions; cool-off questions and closure (see Figure 1).

The interview schedule was piloted within the project team, was reviewed by an external advisor who has considerable expertise in conducting RCTs, and then was reassessed through discussion among the research team after the first two interviews. 


\section{INSERT FIGURE 1 HERE}

\section{Data collection}

The first two interviews were conducted face-to-face, with the remaining 10 conducted by telephone or Skype (see Figure 1). These three modalities were used to facilitate data collection across multiple countries, multiple time zones and to ensured maximum convenience for the participants. Each interview lasted approximately 60 minutes and, with the participants' consent, was audio recorded and then transcribed. All audio recordings were made with an Olympus Digital Dictaphone recorder. All transcriptions were anonymised prior to analysis.

\section{Rigour}

Ensuring rigour within the investigatory process of a qualitative study is essential to the validity and credibility of the findings (Stratford \& Bradshaw, 2016). Guba and Lincoln (1989) propose that, rather than focusing on reliability and validity, as in quantitative research, qualitative studies should focus on trustworthiness. Commonly used domains to define trustworthiness include: credibility, transferability, dependability, and confirmability.

To ensure credibility, i.e. confidence that the findings are true and accurate, a number of steps were taken. To familiarise with the subject matter under investigation, a systematic review of the literature was completed. One of the authors (PM) conducted the interviews. The transcriptions were completed by a third party outside of the research team and were then checked for accuracy by $\mathrm{PM}$ - thus achieving prolonged engagement with the data. The order of the questions was strategically designed to build trust with the participants. Objectivity in the coding process was checked by a second reviewer (LT) agreeing coding definitions and application throughout the 
coding process. Triangulation of the data was ensured by including participants from different professional backgrounds, different amounts of experience in the field and from different countries.

To ensure transferability: we used purposive and opportunity sampling, involving participants from six countries, across three continents and from different professional backgrounds. We also paid particular focus in monitoring saturation. Contradictory cases were sought throughout the coding process and are accounted for in the Findings section below.

To ensure dependability and confirmability: we ensured that the study design and sampling frame was clearly defined when applying for ethical approval; that a semi-structured interview schedule was designed, tested and used for all of the interviews; and that the framework for analysis was defined a priori.

\section{Saturation}

Saturation is an important, yet hard to define, issue in qualitative or mixed-methods studies (Fusch $\&$ Ness, 2015). There are no distinct guidelines on how to assess when saturation has occurred.

Dibley (2011) discussed the nature of data that is thick in quantity, and rich in quality. Rich data is detailed, many-layered and intricate whilst thick data is plentiful.

In this study we considered saturation at the micro and macro levels. At a micro level, at the end of each interview we asked the respondents if they could think of any further barriers/challenges that had not been discussed within the preceding questions. At a macro level, we considered saturation as being reached when we had obtained data which was rich, thick, and adequate to allow us to answer our research questions. Due to the rich number of themes and sub-themes, and 
the thick number of sources and amount of references for each theme, we concluded that we had collected enough data to answer the research question and that we had reached saturation.

\section{Analysis}

The interview recordings were transcribed, anonymised and then analysed using a data management tool - NVIVO (QSR International Pty Ltd. Version 11, 2017). The analysis was conducted using a 6-phase Thematic Analysis Approach (Braun and Clarke, 2006). Phase 1 entailed familiarisation with the data through repeated listening to the audio recordings and reading the transcripts. In Phase 2 a series of initial codes were generated from the transcripts. In Phase 3 relationships between the codes were examined and a series of overarching themes and sub-themes were identified. In Phase 4 the relationships between and within the various themes, sub-themes and codes were reviewed and finalised. In Phase 5 the themes and sub-themes were defined and named. Phase 6 in the Braun and Clarke model is the presentation of the findings.

\section{Ethical Approval and Consent}

Ethical approval was provided by the Ulster University Ethics Filter Committee in Northern Ireland. Each potential interviewee was sent an invitation e-mail along with a participant information booklet which detailed the purpose of the study and a consent form. Written informed consent was obtained from the participants.

\section{Findings}

\section{Main themes:}

From the initial inductive analysis of the transcriptions, a total of 79 codes were identified. From these codes, eight main themes emerged relating to barriers concerning 1) participant co- 
morbidities, 2) participant ability levels, 3) ethics and consent, 4) the RCT methodology, 5) gatekeeping, 6) staff turnover, 7) lack of technical understanding and 8) attitudes and perceptions.

From these eight themes, three overarching higher-order themes were identified which relate to 1) Participant Factors, 2) Design Factors, and 3) Systems Factors. The sub-theme of Attitudes and Perceptions was found to influence the three main themes and was itself influenced by the 'Lack of Technical Understanding' sub-theme (see Figure 2).

\section{INSERT Figure 2: Main themes and sub-themes arising from the data}

\section{Theme 1: Participant Factors}

\subsection{Varying Ability Levels}

Impact on Recruitment: People with ID display a wide range of cognitive, functioning, and communication 'ability' levels. This can pose many challenges to recruitment and data collection attempts. Information leaflets targeted at a certain communication/IQ level may be off-putting to those with a different ability level. This means that researchers need to be mindful of the resources they use to attract potential participants.

$$
\begin{aligned}
& \text { "first of all population, it's very heterogeneous, the IQ varies a lot," } \\
& \text { (06, Academic, Spain) }
\end{aligned}
$$

\footnotetext{
Although the main focus of this study is on adult trials, one researcher remembered a challenge that he faced in a previous trial with children. The recruitment materials and resources that they used contained images of children who appeared to be more disabled than some potential participants. For some potential participants this was off-putting and impacted on recruitment.
} 
"the kids in the state schools looked at these pictures and said 'that is not us' so we don't want to be part of it, so be aware of your imagery and discrimination." (04, Medic, Australia)

Clearly then, it is important that images in promotional materials are relevant to potential participants. With ID trials, this may mean having a broad range of resources appropriate for the range of ability levels within the sample frame.

Understanding of Research Concepts: Even when researchers do manage to match their information resources to the ability levels of the participants, they still have to communicate vague concepts that are often not within common parlance. There was a common perception amongst interviewees that even other professionals within research and clinical fields do not fully understand some of the central underpinnings of the RCT methodology (such as randomisation). And yet, due to good practice guidelines, the trialists have to find ways to explain these complex or vague concepts to people with cognitive or intellectual disabilities.

"if you have learning disabilities or an intellectual disability, it is quite difficult to understand the concept of research. Not at least if it's some randomised control trial and that's not very easy to understand for other people either. “ (10, Public Health, Sweden)

Mandatory Reporting: For a number of reasons, people with ID are seen as vulnerable adults within research circles. This may in part be due to the various cognitive and life difficulties that they experience, and it could also be due to past incidents of abuse within research activities. 
"Sometimes people are worried about this population, you know the vulnerabilities" (01, Psychiatrist, UK)

Unlike conducting trials with non-disabled adults, ID researchers are more likely to interact with participants' home lives, participants' relatives and their professional support staff. As such, they will have access to information about participants that other researchers may never encounter in mainstream trials. This may have implications for the uncovering, reporting and management of instances of neglect or abuse. Thus, it may pose a challenge for trialists who are more used to working with non-disabled adults.

"people [who] are in circumstances which you think are really difficult, ... working out when to disclose what they are or when to contact services and say "this person is in real trouble, you really need to do something sooner rather than later”. (03, Public Health, UK)

\subsection{Co-Morbidities}

One of the core principles of a randomised control trial is that a sample is tested which is representative of the target population. Obtaining such a representative sample may be a major challenge with people with ID for two reasons; firstly, because the term ID covers such a range of disorders with a myriad of causal factors, and secondly because co-morbidity is a common occurrence with people with ID. Therefore, obtaining a truly representative sample whilst protecting the internal validity of a trial can be a significant challenge. 
“It's a real issue with people with learning difficulties who don't usually have a pure condition, there's a lot of multiple issues and multiple conditions in people's lives." (03, Public Health, UK)

\section{Theme 2: Design Factors}

\subsection{Ethics and Consent}

In eleven interviews the processes of obtaining ethical approval and consent were noted as presenting methodological or practical challenges.

Ethics: Over half of the interviewees discussed challenges or difficulties with the ethics process. It would appear from the interviews that there is a large degree of variability in the interactions between researchers and ethics committees, especially when conducting multi-centre studies or multi-jurisdiction studies. Given that multi-venue studies are a common feature for many ID-trials attempting to achieve adequate sample size, obtaining ethical approval across many sources can be a significant practical barrier. Although this may be the case with any large trial, it would appear that more stringent safeguards are often put in place when participants have an ID.

"there are big differences between different ethic committees in terms of how they approach these kinds of issues and I think that some are much more understanding and much more able to deal with the issues in this population and others and therefore the processes is much smoother than in some ethical committees." (01, Psychiatrist, UK) 
"I think it's more problematic if you've multiple ethics committees"

(04, Medic, Australia)

Half of the interviewees expressed a level of frustration with the process of obtaining ethical approval from various ethics committees.

"we were declined approval on the basis that it was unethical to randomise people and that was an ethics committee, a social work ethics committee."

(09, Psychiatrist, UK)

"I think sometimes it's a lack of knowledge, like [implying] that these people can't be interviewed“" (07, Psychologist, USA)

However, the experience of frustration with ethics committees was by no means universal. Almost half of the interviewees reported a collegial working relationship with ethics committees and saw them as a source of expertise, guidance and providing a system of 'checks and balances'.

"we have worked with some ethics committees that were extremely good and have made valuable suggestions of improvement and have been very responsive and quick to deal with the issues as well. I think generally speaking most ethics committees are hopefully like that but also I have unfortunately had an experience with one or two that have been less helpful...” (01, Psychiatrist, UK) 
"not getting approval means that you have done something that is not right .... I found them very, very helpful and it is usually when I have made a mistake when they have come back” (02, Psychiatrist, UK)

Consent: Having a robust pre-defined process of assessing consent is critical when conducting trials with people with ID. Six interviewees reported their experiences and thoughts on this topic, noting that due to the variability in IQ and communication levels within the population, assessing ability to provide consent can be challenging.

"because this is a vulnerable population there's extra things that you have to do to demonstrate that you are truly getting consent and that completely makes sense " (04, Medic, Australia)

Explaining research purpose and process to a disabled sample, and then assessing their understanding, can be complex and challenging. The need to ensure that participants make an informed choice regarding consent is readily acknowledged, however there is also the possibility that the level of participant understanding that is required for an ID trial is not always required for non-disabled populations.

"It's making sure that you have a proper direct way of assessing mental capacity or capacity to consent to the study...I wouldn't want to set the bar higher for people with learning disabilities compared to anyone else, cause I think there is sometimes that tendency." (03, Public Health, UK) 
It should be noted however, that not all interviewees considered the process of assessing and obtaining consent as a methodological challenge.

"I've never really come across consent as being a problem. It takes time, obviously, because you have to do it properly." (09, Psychiatrist, UK)

\subsection{The RCT Methodology}

A strong theme that emerged from the interviews is the potential for an RCT to become too complex and unwieldy. This can occur due to a number of factors, including: levels of bureaucracy inherent in the systems within which the research is occurring; the amount of burden placed upon the various stakeholders; and the range of needs and agendas of each of the stakeholders. Of course, this could be true for any RCT, however the interviewees highlighted a number of key issues specific to conducting trials with people ID.

Bureaucracy: Unlike many non-disability trials, researchers conducting ID trials are rarely permitted to recruit directly and must instead depend upon the goodwill of other agencies and professionals to recruit - this can have variable implications as the systems within which these agencies operate can often be extremely complex.

"we have had situations where there was an agreement, there was the ethics, all of that, and then there was a problem within social care. "We want another ethical review from our own committee"..” (02, Psychiatrist, UK)

Burden: Ensuring that participant burden does not encourage drop out is an important factor in any trial. Participants with ID often rely upon a range of family members and staff for their 
recruitment, participation, and data collection. Mulhall et al (2018) termed these 'co-participants', noting that for every 'participant' in an ID trial, researchers, in reality, must recruit a wider range of 'co-participants', each with the potential to introduce their own source of bias and each with their own agendas, pressures, stresses and limitations. Thus, with ID trials, or when including people with ID in a trial, the detrimental impact of burden can grow exponentially. This must be carefully planned for, and managed.

"if you are asking people to commit to a study over a long period of time, that is a big ask, especially if the participant has other day opportunities to attend which they more than often don't wish to miss out on." (12, Psychologist, UK)

"the way to address that is to make sure the burden on participants are minimised so we try and make sure that we don't overburden participants or carers with extremely lengthy assessments or very frequent research assessments" (01, Psychiatrist, UK)

Funders limiting scope of trials: Some interviewees noted that the process of trial design was not just based upon scientific questioning but can also be influenced by certain stakeholders, particularly funders.

"some of the major decisions about the design of the study were kind of made in negotiation with the funders." (03, Public Health, UK)

"However, the funder, in their wisdom, felt that we should start with people with more mild disabilities and they made it a pre-condition" (08, Psychologist, UK) 
Whilst there was an acknowledgement amongst interviewees that ID RCTs can be "expensive and difficult to run", there was a perception that some funders "have a limited knowledge of this population", do not appreciate the complexities of conducting ID trials and are of the opinion that "this isn't a group that's worthwhile investing research funding in".

“it's very hard to make funders who have no understanding of this population aware of the complexities involved in getting meaningful data" (04, Medic, Australia)

However, this perception was not universal, and it was noted that not all trials applications experience the same reception.

\section{Theme 3 Systems Factors}

\subsection{Gatekeeping}

As noted above, rarely can ID researchers recruit directly to participants and therefore must recruit via other professionals or guardians. The process of gatekeeping was one of the most pertinent issues being mentioned, with eight interviewees discussing it directly. This is hardly surprising as the decisions made by gatekeepers have a direct impact on recruitment levels and therefore, potentially, the successful completion of the trial. The factors that influence a gatekeeper's decision-making process may overlap with all of the previously discussed barriers. Thus, navigating and negotiating through this multi-layered process of gatekeeping poses significant barriers for ID researchers. 
"recruiting is a big issue and that's probably given the fact that we rely heavily on the primary carer and also community learning disability teams ... they are a major influence in the decision making for the person with a learning disability," (12, Psychologist, UK))

“Sometimes it's the support staff that don't have time, or can't be bothered, .....or are frightened of not knowing if they're breaking the rules, not really understanding issues around people who can't consent for themselves. [are] worried they'd do something wrong. “( 09 , Psychiatrist, UK)

There was a perception among some interviewees that not being able to recruit directly poses many unnecessary challenges for ID researchers. This can lead to unnecessarily repetitious assessment and scrutiny regarding a trial's design, ultimately leading to delays, changes to protocols and resources, increased expenses and potentially lead to a trial not proceeding.

"some local services felt that they had to go through many of the details of the study including information sheets and consent procedures and consent forms and then they want you to change things at local level " (01, Psychiatrist, UK)

Gatekeepers Decision Making: It could be argued that each of the layers of gatekeepers provides increasing levels of protection for the person with ID. At each stage gatekeepers are meant to make decisions based upon the best interests of the individual concerned. This is clearly stipulated in legislation such as the UK's Mental Capacity Act (2005). The perception among some interviewees was that this does not always happen.

There was a perception that some of the decisions made by gatekeepers were not always based upon the best interests or wishes of the person with ID, but rather were based upon a series of 
other factors: including staff having limited time, the pressures of already heavy workloads and being "worried they'd do something wrong. "

Not all experiences working with gatekeepers were negative, in terms of staff resistance to research, some found that "In the beginning, they were a little bit afraid it would take too long a time, but when we explained the intervention, I think they were actually positive to the intervention" [10, Public Health, Sweden]. There was also an appreciation amongst the interviewees that when a person with ID participates in a study, it may have real implications for the professional staff. There was also an acknowledgement that although staff may in fact be supportive, the systems within which they work may not always be conducive to research activities.

\footnotetext{
"with staff members as well, they may only be working certain hours with that particular person and it may not be conducive for them to be involved in the study over such a prolonged period of time and make that commitment." (12, Psychologist, UK)
}

There was a perception among some interviewees that with the evolution of services provided to people with ID, improved ethical standards of research practice, coupled with a move to generate greater levels of life-choice autonomy for people with ID, the current level of protectionist measures may be becoming counter-productive. Rather than protecting the rights of people with ID from research activities, they may in fact deny the opportunity to people with ID, particularly those with mild to moderate ID, to make their own decisions.

"you can disadvantage people by excluding them from research, as opposed to making them vulnerable by including them" (cited above) (08, Psychologist, UK) 
“while they say they're protecting a proportion of the population, in fact they're limiting their ability to create an evidence base that would actually help them."

(05. PAM, Australia)

\subsection{Staff Turnover}

The influence that systemic factors have on participation can be significant. With disabilityfocused trials, staffing levels, workload, and staff turnover can have a detrimental impact on recruitment, retention, and data collection.

"for us it was the staff attrition, therapist attrition was a big issue..... we had a very high turnover of staff, so in terms of the number of therapists that we had to train - it was actually a very large number of therapists who then saw a very small number of people each,“(03, Public Health, UK)

"staff turnover is always a bit of an issue." (08, Psychologist, UK)

\subsection{Lack of Technical Understanding}

There was a belief amongst some interviewees that a lack of understanding regarding the RCT process, and utility, permeates across many researchers, clinical staff, participants and carers.

“most community people don't understand RCTs or the value of RCTs." (07, Psychologist, USA)

"I think that people don't always understand how medication trials work in this population." (01, Psychiatrist, UK) 
This lack of understanding can have a huge impact on the success of a trial at all stages; from planning, to recruitment and then reporting. It would appear that one of the major sources of misunderstanding was concerning the process of randomisation.

\footnotetext{
"when you start to talk about trials, people don't really like the idea of randomisation" (01, Psychiatrist, UK)
}

This lack of understanding regarding RCTs and randomisation may be a major contributor to the reluctance of gatekeepers to facilitate, encourage or allow participation in an RCT.

\author{
"I think that carers and key workers, health care professionals, feel that it is wrong \\ to deprive a person of what they see as a needed treatment." \\ (12, Psychologist, UK)
}

\title{
Attitudes and Perceptions
}

As well as acknowledging many of the practical barriers that get in the way of recruitment to trials, there was also an understanding amongst interviewees that not everyone is interested in participating in research and that lack of desire to partake must be respected - this refers to the people with ID, their relatives, and support staff. Eight of the interviewees mentioned the issue of attitudes and how this may impact on a trial.

"you have to accept that not everyone will see research as being helpful but also we had loads of feedback to say that people did find research helpful because they have got something" (02, Psychiatrist, UK) 
"Staff and relatives are frightened for letting people with ID be in research."

(11, Medic, Sweden)

Staff attitudes towards research is of obvious importance and has been discussed previously in the literature (Oliver-Africano et al., 2010). An interesting theme that emerged in the interviews that is not so well documented is the interviewee's perceptions regarding the attitudes of funders, and of the wider research community towards people with ID.

Funders: There was a perception among some interviewees that not all funders consider trials with this population to be economically viable or worth investing in. Some thought that funders were unfairly comparing population size, population needs and trial costs with other disabilities/health conditions and making their decisions based upon financial outcomes rather than equality of access to research.

"really what they're saying, is that this isn't a group that's worthwhile investing research funding in." (05, PAMS, Australia)

Scientific community: The perceived attitudes of the wider scientific community were also of concern to some of the interviewees. There was a perception that some researchers are unsure of the benefits of conducting research in this area and that this uncertainty can lead, either directly or indirectly, to bias and discrimination.

"I honestly believe that the discrimination against this population is so deep and so embedded ... even [amongst] so-called scientists, who are very objective. “" 
(04, Medic, Australia)

It is possible that the negative attitudes encountered by ID trialists are not founded directly in prejudice but rather it points to a wider problem which is a lack of understanding, described above, concerning the needs and abilities of people with ID, the RCT methodology, and the utility of RCTs with this population.

\section{Discussion}

Traditionally, a common note in many systematic reviews in the ID field is the lack of ID-RCTs. Although there have been a number of guidelines produced to support the development and conducting of RCTs (MRC guidelines, CONSORT etc), these guidelines have focused mainly on procedural, legal and reporting issues. To date there has been little guidance on the practicalities or realities (challenges and barriers) of conducting RCTs with hard to include populations, in this case people with ID, and this paper adds to the international knowledgebase in this field.

As discussed above, there have been a few 'methods' papers which have discussed specific challenges faced whilst conducting specific ID trials however, the extent to which these challenges are commonplace has not been previously explored. Thus, the findings from this study are the first to document, collate and share the experiences of an international group of ID-RCT researchers.

\section{Confirmation of previously cited challenges}

From the analysis of the data in this study eight main themes emerged relating to barriers concerning 1) participant co-morbidities, 2) participant ability levels, 3) ethics and consent, 4) the RCT methodology, 5) gatekeeping, 6) staff turnover, 6) lack 
of technical understanding, and 7) attitudes and perceptions. Some of these findings confirm that challenges that were previously cited such as staff work load, staff turnover and complex agency structures (Lennox et al., 2005); excessive governance structures (Leeson and Tyrer, 2013); heterogeneity of participants (Jacquerment et al., 2014); the reliance on organisations which may fear 'outsiders' (Siegel \& Ellis, 1985) are in fact common experiences amongst ID-RCT researchers.

\section{Contribution of new knowledge}

The findings of the current study also highlight a number of additional methodological and practical challenges not previously discussed in the Mulhall et al. (2018) systematic review or the wider ID-RCT literature.

Heterogeneity of participants: Although the heterogeneity of ID populations has been noted before in the ID literature (for example Jacquermont et al., 2014), the practical implications and barriers that it may cause for trials are not so widely documented. For example, information leaflets targeted at certain communication/IQ levels may be off-putting to those with a different ability level. Therefore, it is important that researchers are mindful of the resources they use to attract potential participants and to explain the research project. This may necessitate different ability-specific versions of information sources and consent forms (such as those used HornerJohnson et al., 2011), using accessible language and/or images (such as used by Turk et al., 2010), and making careful adaptations to program content and design (such as Melville et al., 2015).

Heterogeneity of agencies: The negative impact of heterogeneity is not limited to that of the participants with ID. The variety of service provision agencies, the myriad governance structures, the various clinical and social care staff involved and the needs of family carers, all add additional layers of complexity that pose challenges not only to obtaining trial approval but also in ensuring 
protocol adherence. The true extent of this challenge has not been previously discussed in the IDtrials literature, nor has the possibility of agency-specific variance regularly been assessed in the analyses of many trials.

Co-Participants: It is clear from the findings of this study that people with ID rely heavily upon a range of sources (family members, professional staff, service provision agencies etc) not only for support with daily living (Brown et al., 2017), but also for participation in research studies.

Consequently, not only do ID trialists have to recruit a given number of participants, they have to 'recruit' a potentially larger number of support-providing gatekeepers. Mulhall et al. (2018) termed these support providers 'co-participants' as each participant with ID relies heavily on the 'co-participants' for their recruitment, retention and data collection.

Establishing systems for identifying, and approaching, potential participants is vital for any trial. Accessing participants was a commonly reported challenge and appears to be a common feature of many ID trials, with most ID trials failing to recruit more than 100 participants (Mulhall et al., 2018) and yet trials' reports rarely specify accessing participants as being an actual challenge. Again, this suggests that there are a range of barriers and challenges that are not being shared in the trials literature.

Ethics: A finding that has not been previously cited in the international ID-RCT literature is reports of frustration and inconsistent experiences whilst applying for ethical approval for an IDRCT, particularly when planning for a multi-centre or multi-jurisdiction trial. Ethical standards are seen as central to high quality research practice (Medical Research Council, 2005) and many journals now require a statement that ethical approval was obtained, and that informed consent was obtained from participants, for any study involving human participants. However, most of 
these statements appear as a single sentence in a trial report, which is not reflective of the amount of time or effort that is expended in obtaining the ethical approval, nor the barriers and challenges experienced in the process.

It would appear from the findings of this study that there is more work to be done to ensure clarity and consistency regarding implementation of relevant legislation and to aid the development of a greater collegial partnership between ethics committees/governance boards, gatekeepers and trials researchers.

As frustrating as the experience of applying for ethics had been for some of the interviewees, it was also appreciated that there had in the past been instances of poor practice during research activities with people with ID, and that this justified the need for measures to improve practice and ensure that such occurrences would not be repeated. However, rather than protecting people with ID from dubious research activities, it was reported by some interviewees that such measures are now removing the ability of people with ID, particularly those with mild to moderate ID, to make their own decisions, specifically about participation in research. So, whilst acknowledging the need for ethical guidelines and the protection of vulnerable people, it is also important that such guidelines do not inadvertently exclude people with ID from having the opportunity to take part in research.

With the evolution of services and support provided to people with ID, and improved ethical standards of research practice, coupled with a move to generate greater levels of life-choice autonomy for people with ID, the current level of protectionist measures may be becoming counter-productive. 
"You can disadvantage people by excluding them from research, as opposed to making them vulnerable by including them." (Psychologist, UK)

The relevance, and importance, of this finding extends well beyond the field of ID-RCTs and suggests the need for a wider debate regarding the relationship between people with ID, how best the research community can meet their needs, how ethics and governance structures can work in partnership with researchers, and whether the current systems are fit for purpose given the societal and service-provision changes that have occurred over the past few decades.

Attitudes and Perceptions: Perhaps the most striking finding of this study was the experiences of the researchers concerning the attitudes and perceptions of others. There is growing evidence that healthcare staff are generally favourable towards research activities (Caldwell et al., 2017). However, the findings from this study suggests that such positivity for research does not always extend towards ID-RCTs. It would appear that many people (clinical, research and lay) still hold negative attitudes towards RCTs, and particularly with ID populations. We have documented that some interviewees reported experiencing prejudiced attitudes towards people with ID and towards the utility of conducting RCTs with these populations, particularly from funders. Of course it is possible that the foundation for this is not prejudice against people with ID but rather a lack of agreement concerning the cost-benefit ratio or utility of ID-RCTs. To the best of our knowledge this is a new finding and one that has not been discussed previously in the literature.

The attitudes and prejudices reported in this study may also be explained by the desire of those involved to protect people with ID, coupled with a lack of technical understanding about the fundamental principles and methodology of an RCT. It was surprising, for example, that some clinical and academic staff did not appear to understand the mechanism, or legality, of 
randomisation. To the best of our knowledge this fundamental lack of understanding within the wider academic and professional fields, about core aspects of RCT methodology, and its impact on the trial process, has not been highlighted previously. Regardless of the root causes of these attitudes, they can be detrimental to a trial at all levels, from concept design through to recruitment, data collection, reporting in the literature and influencing practice. There is clearly a challenge here for RCT researchers in dispelling what appears to be very prevalent myths and misunderstandings about the RCT methodology in the ID field.

The majority of the challenges and barriers found in this study do not appear to be bound within specific jurisdictions, however a small number of international nuances are of note. In terms of seeking ethical approval for larger trials, the UK's governance system was seen as problematic when conducting multi-country studies. A psychiatrist from the UK described a trial for which "all the other ethical committees in the rest of the world had approved the study but in the UK we ran into difficulties"

However, it should be noted that the UK's Research Ethics Service have made significant changes in the mechanisms for seeking ethical approval (https://www.hra.nhs.uk/about-us/committeesand-services/res-and-recs/). On the other hand, countries such as Australia often have a relatively small number of providers for a relatively large geographical spread. This has obvious advantages when it comes to recruitment as fewer agencies may result in fewer ethical application processes, although it may add additional practical challenges, including costs for travel.

Most of the jurisdictions represented in this study do not have a register of people who have been diagnosed with an ID. Where registers are held, accessing them can be difficult for researchers (due to the sensitive nature of the data being held) and consequently, in practice, it was reported 
by one of the interviewees that their use is often avoided by researchers, if possible. It would appear that accessing registers of potential participants might be easier in some states in the US where registers are available for recipients of certain services, benefits or waivers, but this is anecdotal and a review of the existence of registers across jurisdictions would be a useful addition to the literature in this field.

As well as discussing the various challenges that they had experienced, the interviewees also displayed a large degree of self-reflection. The final challenge presented in this paper is a challenge to ID-RCT researchers themselves:

"I think a well-written trial gets funded and I think we do have a problem in our field, in that some of the funding applications that are made are just poor quality, and that's because we're a slim research field." (09, Psychiatrist, UK)

\section{Strengths and Limitations of the study}

The authors acknowledge a number of potential limitations with the current study. The sample of participants was drawn from researchers experienced in conducting ID-specific trials and did not include researchers from outside the ID field. However, the study's primary focus was conducting RCTs within the ID field and, as discussed in the Introduction, many mainstream trials regularly exclude people with ID (either directly or indirectly) (Feldman et al. (2014). Future studies examining if RCT researchers face similar challenges and barriers with other cognitive disability populations may shed additional light on this area.

Another potential limitation of this study is the sample size, however, we have shown that saturation was reached and, although small, the sample is representative of the international IDRCT field. There was a larger portion of the sample from the UK, however this is not surprising 
given that the UK has a number of specialist ID services and a number of researchers from the UK have been at the forefront of developing ID-RCTs. The ID-RCT field is relatively new and still developing, and those involved in this study are at the cutting edge of this development. The sample drew upon a portfolio of professional backgrounds, from a range of countries, with a considerable number of years' experience conducting some of the leading clinical trials in this field. This is the first time that an expert panel of ID-RCT researchers have been interviewed to explore this topic.

\section{Conclusion}

This study highlighted a number of practical and methodological barriers and challenges to conducting RCTs with adults with ID. Some of the barriers are related to the RCT methodology itself, others are specific to the population of interest, to the uniqueness of the systems within which the research take places and others are related to the attitudes and perceptions of the various 'stakeholders' in the research process. Although some barriers such as the identification of potential participants have nuanced differences across various jurisdictions due to different service provision models, the majority of barriers and challenges appear to be universal. As such, the results of this study are of international significance. Due to the overlap in sequalae between ID and other cognitive disabilities, we propose that the findings of this study are also of benefit to researchers conducting trials with other disabled populations (such as those with dementia, autism, acquired brain injury) where ability to participate, or to provide consent, may require additional support. 


\section{References}

Aarons, G. A., Hurlburt, M., \& Horwitz, S. M. (2011). Advancing a conceptual model of evidence-based practice implementation in public service sectors. Administration and Policy in Mental Health and Mental Health Services Research, 38(1), 4-23.

Bonevski, B., Randell, M., Paul, C., Chapman, K., Twyman, L., Bryant, J., ... \& Hughes, C. (2014). Reaching the hard-to-reach: a systematic review of strategies for improving health and medical research with socially disadvantaged groups. BMC medical research methodology, 14(1), 42.

Bosco, A., Paulauskaite, L., Hall, I., Crabtree, J., Soni, S., Biswas, A., ... \& Crawford, M. J. (2019). Process evaluation of a randomised controlled trial of PBS-based staff training for challenging behaviour in adults with intellectual disability. PloS one, 14(8), e0221507.

Braun, V. \& Clarke, V. (2006) Using thematic analysis in psychology. Qualitative Analysis in Psychology, 3:2, 77-101.

Brown, C. H., Curran, G., Palinkas, L. A., Aarons, G. A., Wells, K. B., Jones, L., ... \& Tabak, R. G. (2017). An overview of research and evaluation designs for dissemination and implementation. Annual review of public health, 38, 1-22.

Brown, M., Taggart, L., Karatzias, T., Truesdale, M., Walley, R., Northway, R., ... \& Davies, M. (2017). Improving diabetes care for people with intellectual disabilities: a qualitative study 
exploring the perceptions and experiences of professionals in diabetes and intellectual disability services. Journal of Intellectual Disability Research, 61(5), 435-449.

Button, K. S., Ioannidis, J. P., Mokrysz, C., Nosek, B. A., Flint, J., Robinson, E. S., \& Munafò, M. R. (2013). Power failure: why small sample size undermines the reliability of neuroscience. Nature Reviews Neuroscience, 14(5), 365.

Caldwell, B., Coltart, K., Hutchison, C., McJury, M., Morrison, A., Paterson, C., \& Thomson, M. (2017). Research awareness, attitudes and barriers among clinical staff in a regional cancer centre. Part 1: a quantitative analysis. European journal of cancer care, 26(5), e12434.

Camerer, C. F., Dreber, A., Holzmeister, F., Ho, T. H., Huber, J., Johannesson, M., ... \& Altmejd, A. (2018). Evaluating the replicability of social science experiments in Nature and Science between 2010 and 2015. Nature Human Behaviour, 2(9), 637.

Connell, J., Carlton, J., Grundy, A., Buck, E. T., Keetharuth, A. D., Ricketts, T., ... \& Brazier, J. (2018). The importance of content and face validity in instrument development: lessons learnt from service users when developing the Recovering Quality of Life measure (ReQoL). Quality of Life Research, 27(7), 1893-1902.

Cooper, S. A. (2017). The prevalence of physical conditions and multi-morbidity in a cohort of adults with intellectual disabilities, with and without Down syndrome. Cross-sectional study. $B M J$ Open. 
Department for Constitutional Affairs. (2007). Mental capacity act 2005: code of practice. HM Stationery Office.

Dibley L (2011) Analysing narrative data using McCormack's Lenses. Nurse Researcher. 18, 3, 13-19.

Duncombe, D. C. (2018). A multi-institutional study of the perceived barriers and facilitators to implementing evidence-based practice. Journal of Clinical Nursing, 27(5-6), 1216-1226.

Feldman, M.A., Bosett, J., Collet, C. and Burnham-Riosa, P., (2014). Where are persons with intellectual disabilities in medical research? A survey of published clinical trials. Journal of Intellectual Disability Research, 58(9): 800-809.

Ford JG, Howerton MW, Lai GY, Gary TL, Bolen S, Gibbons MC, et al. (2008) Barriers to recruiting underrepresented populations to cancer clinical trials: a systematic review. Cancer. 112: $228-242$.

Freedman, L. P., Cockburn, I. M., \& Simcoe, T. S. (2015). The economics of reproducibility in preclinical research. PLoS biology, 13(6), e1002165.

Fusch, P. I., \& Ness, L. R. (2015). Are we there yet? Data saturation in qualitative research. The qualitative report, 20(9), 1408.

Glover, M., Kira, A., Johnston, V., Walker, N., Thomas, D., Chang, A. B., ... \& Brown, N. (2015). A systematic review of barriers and facilitators to participation in randomized controlled trials by 
Indigenous people from New Zealand, Australia, Canada and the United States. Global health promotion, 22(1), 21-31.

Guba, E. G., \& Lincoln, Y. S. (1989). Fourth generation evaluation. London: Sage.

Hassiotis, A., Poppe, M., Strydom, A., Vickerstaff, V., Hall, I., Crabtree, J., ... \& Biswas, A. (2018). Positive behaviour support training for staff for treating challenging behaviour in people with intellectual disabilities: a cluster RCT. Health Technology Assessment, 22(15), 1-110.

Hastings, R., (2013). Running to catch up: rapid generation of evidence for interventions in learning disability services. British Medical Journal, 203, 245-246

Heiat, A., Gross, C. P., \& Krumholz, H. M. (2002). Representation of the elderly, women, and minorities in heart failure clinical trials. Archives of internal medicine, 162(15).

Hunter, R. M., Vickerstaff, V., Poppe, M., Strydom, A., King, M., Hall, I., ... \& Hassiotis, A. (2020). Staff training in positive behaviour support for behaviour that challenges in people with intellectual disability: cost-utility analysis of a cluster randomised controlled trial. BJPsych Open, 6(2).

Horner-Johnson, W., Drum, C. E., \& Abdullah, N. (2011). A randomized trial of a health promotion intervention for adults with disabilities. Disability and health journal, 4(4), 254-261. 
Jacquemont, S., Berry-Kravis, E., Hagerman, R., Von Raison, F., Gasparini, F., Apostol, G., Ufer, M., Des Portes, V. and Gomez-Mancilla, B., (2014). The challenges of clinical trials in fragile X syndrome. Psychopharmacology, 231(6): 1237-1250.

Jones, E., \& Chaplin, E. (2017). A systematic review of the effectiveness of psychological approaches in the treatment of sex offenders with intellectual disabilities. Journal of Applied Research in Intellectual Disabilities.

Kadam, R. A., Borde, S. U., Madas, S. A., Salvi, S. S., \& Limaye, S. S. (2016). Challenges in recruitment and retention of clinical trial subjects. Perspectives in clinical research, 7(3), 137.

Katkade, V. B., Sanders, K. N., \& Zou, K. H. (2018). Real world data: an opportunity to supplement existing evidence for the use of long-established medicines in health care decision making. Journal of multidisciplinary healthcare, 11, 295.

Koslowski, N., Klein, K., Arnold, K., Kösters, M., Schützwohl, M., Salize, H. J. and Puscher, B., (2016). Effectiveness of interventions for adults with mild to moderate intellectual disabilities and mental health problems: systematic review and meta-analysis, British Journal of Psychiatry, 209(6): 469-474

Leeson, V.C. \& Tyrer, P., (2013). The advance of research governance in psychiatry: one step forward, two steps back. Epidemiology and Psychiatric Services, 22(4): 313-320 
Lennox, N., Taylor, M., Rey-Conde, T., Bain, C., Purdie, D.M. and Boyle, F., (2005). Beating the barriers: recruitment of people with intellectual disability to participate in research. Journal of Intellectual Disability Research, 49(4): 296-305.

McGill, P., Vanono, L., Clover, W., Smyth, E., Cooper, V., Hopkins, L., ... \& Davis, S. (2018). Reducing challenging behaviour of adults with intellectual disabilities in supported accommodation: A cluster randomized controlled trial of setting-wide positive behaviour support. Research in developmental disabilities, 81, 143-154.

Medical Research Council (2005). MRC ethics series: Good research practice (2005). London: Medical Research Council , $2005 ; 1-16$

Melville, C. A., Mitchell, F., Stalker, K., Matthews, L., McConnachie, A., Murray, H. M., ... \& Mutrie, N. (2015). Effectiveness of a walking programme to support adults with intellectual disabilities to increase physical activity: walk well cluster-randomised controlled trial. International Journal of Behavioral Nutrition and Physical Activity, 12(1), 125.

Moore GF, Audrey S, Baerker M, Bond L, Bonell C, Hardeman W, et al., (2015). Process evaluation of complex interventions: Medical Research Council guidance. BMJ. 2015;350:h1258

Mulder, R., Singh, A. B., Hamilton, A., Das, P., Outhred, T., Morris, G., ... \& Lyndon, B. (2018). The limitations of using randomised controlled trials as a basis for developing treatment guidelines. Evidence-based mental health, 21(1), 4-6. 
Mulhall, P., Taggart, L., Coates, V., McAloon, T., \& Hassiotis, A. (2018). A systematic review of the methodological and practical challenges of undertaking randomised-controlled trials with cognitive disability populations. Social Science \& Medicine, 200, 114-128.

Oakley, A., Wiggins, M., Turner, H., Rajan, L., \& Barker, M. (2003). Including culturally diverse samples in health research: a case study of an urban trial of social support. Ethnicity and Health, 8(1), 29-39.

Oliver-Africano, P., Dickens, S., Ahmed, Z., Bouras, N., Cooray, S., Deb, S., ... \& Bhaumik, S. (2010). Overcoming the barriers experienced in conducting a medication trial in adults with aggressive challenging behaviour and intellectual disabilities. Journal of Intellectual Disability Research, 54(1), 17-25.

Prinz, F., Schlange, T., \& Asadullah, K. (2011). Believe it or not: how much can we rely on published data on potential drug targets?. Nature reviews Drug discovery, 10(9), 712.

Robotham, D., King, M., Canagasabey, A., Inchley-Mort, S., \& Hassiotis, A. (2011). Social validity of randomised controlled trials in health services research and intellectual disabilities: a qualitative exploration of stakeholder views. Trials, 12(1), 144.

Robson, C. (2011) Real world research: a resource for users of social research methods in applied settings. Wiley Chichester.

Roland, M., \& Torgerson, D. J. (1998). Understanding controlled trials: What are pragmatic trials?. Bmj, 316(7127), 285. 
Scheifes, A., Stolker, J. J., Egberts, A. C. G., Nijman, H. L. I., \& Heerdink, E. R. (2011). Representation of people with intellectual disabilities in randomised controlled trials on antipsychotic treatment for behavioural problems. Journal of Intellectual Disability Research, 55(7), 650-664.

Siegel, P.S. and Ellis, N.R., (1985). Note on the recruitment of subjects for mental retardation research. American journal of mental deficiency. Cited in Lennox, N., Taylor, M., Rey-Conde, T., Bain, C., Purdie, D.M. and Boyle, F., (2005) Beating the barriers: recruitment of people with intellectual disability to participate in research. Journal of Intellectual Disability Research, 49(4): 296-305.

Stratford, E and Bradshaw, M, (2016) Qualitative Research Design and Rigour, Qualitative Research Methods in Human Geography. Oxford University Press, I Hay (ed), Ontario, Canada, pp. 117-129. ISBN 978-0-19-901090-5

Turk, V., Burchell, S., Burrha, S., Corney, R., Elliott, S., Kerry, S., ... \& Painter, K. (2010). An evaluation of the implementation of hand held health records with adults with learning disabilities: a cluster randomized controlled trial. Journal of Applied Research in Intellectual Disabilities, 23(2), 100-111.

Tyrer, P., Tarabi, S. A., Bassett, P., Liedtka, N., Hall, R., Nagar, J., ... \& Tyrer, H. (2017). Nidotherapy compared with enhanced care programme approach training for adults with 
aggressive challenging behaviour and intellectual disability (NIDABID): cluster-randomised controlled trial. Journal of intellectual disability research, 61(6), 521-531.

Vereenooghe, L. and Langdon, P.E. , (2013). Psychological therapies for people with intellectual disabilities: A systematic review and meta-analysis. Research in Developmental Disabilities, 34(11): pp.4085-4102.

Williams, M. E., Hastings, R. P., \& Hutchings, J. (2020). The incredible years autism spectrum and language delays parent program: A pragmatic, feasibility randomized controlled trial. Autism Research. 


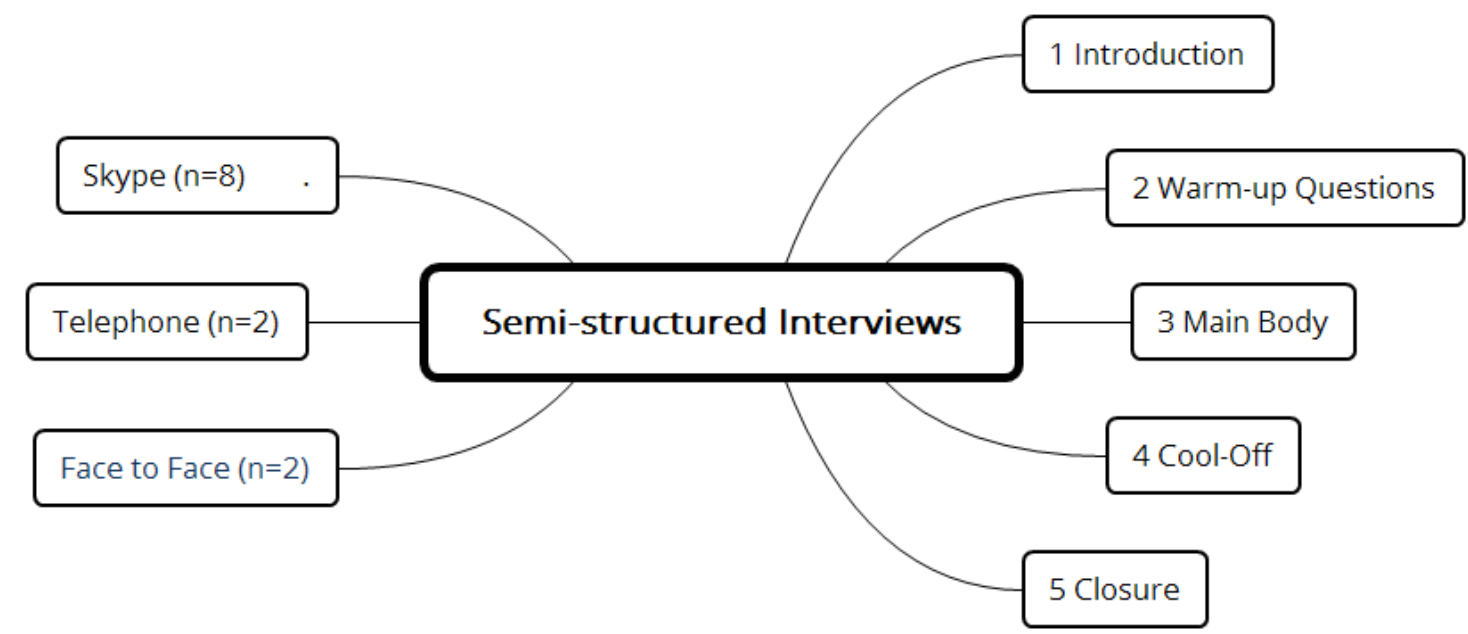

Figure 1: Medium used to conduct the interviews and 5 Stage Interview structure 


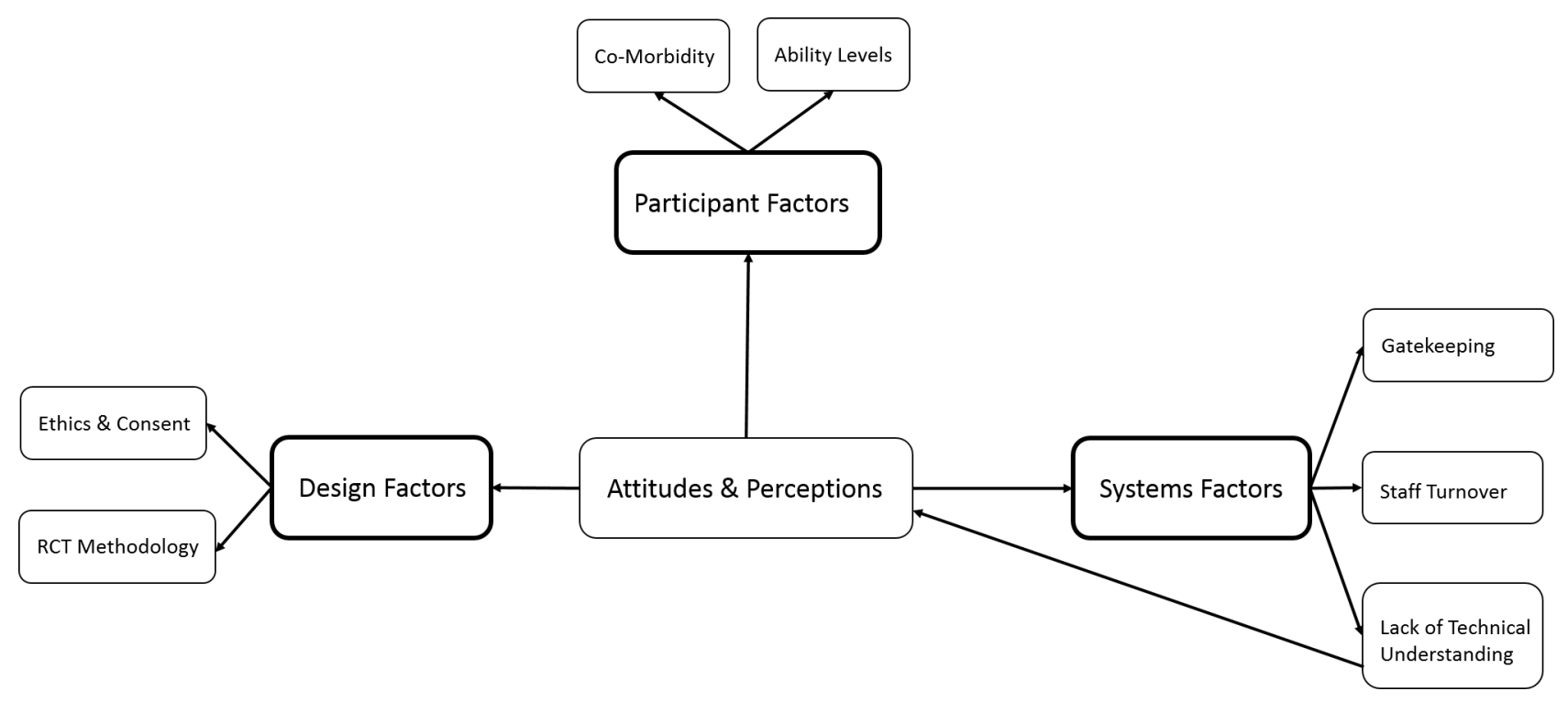

Figure 2: Main themes and sub-themes arising from the data 